\title{
Aktuelle Aspekte der Zeckenassoziierten neurologischen Infektionen Reinhard Kaiser
}

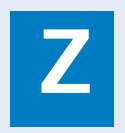

ecken zählen allgemein zu den am meisten verabscheuten Insekten, nicht nur weil sie sich in der Haut des Menschen so festbeißen, dass sie ohne chirurgische Hilfe häufig kaum zu entfernen sind, sondern auch, weil sie Krankheiten übertragen, die zu schweren Beeinträchtigungen und Defektheilungen führen können. Die kürzlich publizierte Beobachtung, dass sie auch den $95^{\circ} \mathrm{C}$ Waschgang überleben können, unterstreicht die hohe Überlebenskunst dieser Spinnentiere, denen auch Temperaturen bis minus $40^{\circ} \mathrm{C}$ wenig anhaben können. Von der Vielzahl der durch sie übertragenen Erkrankungen spielen für den Neurologen in Mitteleuropa epidemiologisch nur zwei eine Rolle: Die Neuroborreliose und die Frühsommer-Meningoenzephalitis. Beide Erkrankungen sind für den Kliniker in den meisten Fällen allein aufgrund ihrer Symptomatik gut zu differenzieren: Bei der FSME steht die schwere Beeinträchtigung des Allgemeinbefindens mit hohem Fieber und Kopfschmerzen ganz im Vordergrund, bei der Neuroborreliose klagt dagegen die Mehrzahl der Patienten über radikulär verteilte Schmerzen, die häufig nicht auf einfache Analgetika ansprechen.

Patienten mit einer Neuroborreliose werden nur selten intensivpflichtig, solche mit einer FSME dagegen häufiger. Die Gründe hierfür finden sich in der unterschiedlichen Pathogenese beider Erkrankungen: Bei der Neuroborreliose entwickeln sich die Symptome aufgrund einer überschießenden Immunantwort auf bestimmte Oberflächenproteine des Erregers (ospA), bei der FSME infolge einer Lyse infizierter Neurone, wobei das Virus eine Prädilektion für die motorischen Hirnnervenkerne, die Vorderhornzellen im Rückenmark, die Purkinjezellen im Kleinhirn sowie Neuronenverbände im Thalamus und die Meningealzellen aufweist. Patienten mit einer FSME können innerhalb weniger Stunden bis ca. 3 Tage seit Erkrankungsbeginn (Fieberanstieg) intubationspflichtig werden, sodass bei entsprechendem Krankheitsverdacht zunächst eine engmaschige klinische Überwachung erforderlich ist. In seltenen Ausnahmefällen kann sich die FSME aber auch als isolierte Myelitis ohne Zeichen einer Meningitis oder Enzephalitis präsentieren (Fauser et al 2007). Bei der Neuroborreliose führen nur die seltene zerebrale Vaskulitis mit Präedilektion für das hintere Stromgebiet, die akute Enzephalitis mit sehr variablem klinischem Bild und die Querschnittsmyelitis gelegentlich zur Intensivüberwachung.

Für die Diagnostik der Neuroborreliose gelten weiterhin die etablierten Kriterien der Serologie und insbesondere der Liquordiagnostik. Die Infektion ist bei passender klinischer Symptomatik wahrscheinlich bei Nachweis spezifischer Antikörper im Serum und zusätzlicher Pleozytose im Liquor (eine Schrankenstörung und intrathekale Immunglobulinsynthese finden sich häufig, aber nicht generell), sicher bei Nachweis einer intrathekalen erregerspezifischen Antikörpersynthese und/oder positiver PCR im Liquor. Der Stellenwert von Antikörpern gegen das vlsE bzw. das C6 Peptid (konservierter Bereich des vlsE) zur Diagnosesicherung wird zurzeit noch kontrovers diskutiert, dagegen konnte das Chemokin CXCL13 wiederholt als Marker einer akuten Neuroborreliose identifiziert werden (Rupprecht et al 2006). Leider ist der entsprechende Test bislang nicht allgemein verfügbar. Für die Therapie der Neuroborreliose ergaben Metaanalysen früherer Studien eine vergleichbare Wirksamkeit von Doxycyclin, Ceftriaxon, Cefotaxim und Penicillin G (Borg et al 2005, Halperin 2007). Dies überrascht zunächst, da Ceftriaxon eine deutlich bessere Liquorgängigkeit aufweist als Doxycyclin, andererseits zeigten retrospektive Analysen aus den 80er Jahren, dass die einfache Neuroborreliose (isolierte Radikulitis) auch ohne spezifische Antibiotikagabe ausheilen kann. Im Falle einer bedrohlichen neurologischen Symptomatik wird man daher aufgrund der exzellenten in vitro und in vivo Daten eher auf Ceftriaxon zurückgreifen, bei einer einfachen Radikulitis ohne dramatische Schmerzen (z. B. isolierte Fazialisparese) kann dagegen gut auf die Wirksamkeit des oral verabreichbaren Doxycyclins vertraut werden. Für das sehr seltene Post-Lyme-Syndrom gibt es bislang keine überzeugenden Hinweise 
für eine Erregerpersistenz oder einen Nutzen wiederholter Antibiotikagaben. Hier steht die symptomatische Therapie im Vordergrund.

Die Diagnostik der FSME gestaltet sich vergleichsweise einfach: Hier genügt der Nachweis spezifischer IgMund IgG-Antikörper im Serum sowie einer Pleozytose im Liquor. Da die FSME die gleiche klinische Symptomatik und dieselben laborchemischen Veränderungen wie die bakterielle Meningitis (CRP-Erhöhung, BSGBeschleunigung, Leukozytose im Blut, granulozytäre Pleozytose im Liquor) aufweisen kann, sollte zur Differenzierung immer die Laktatbestimmung im Liquor erfolgen, welche bei der FSME in der Regel normale Werte ergibt. Die Therapie der FSME ist weiterhin Symptom orientiert, bislang gibt es keine kausalen Therapieansätze.

Verlaufsuntersuchungen von Impflingen haben gezeigt, dass die Abstände für die Auffrischimpfungen bis zu einem Lebensalter von 50 Jahren von bislang 3 auf 5 Jahre verlängert werden können. Bei „vergessener“ Auffrischimpfung kann bei einer kompletten Grundimmunisierung in der Vergangenheit durch eine einzige Boosterimpfung auch nach bis zu 8 Jahren wieder ein vollständiger Impfschutz erreicht werden.

\section{Literatur}

1 Borg R, Dotevall L, Hagberg L, Maraspin V, LotricFurlan S, Cimperman J, Strle F. Intravenous ceftriaxone compared with oral doxycycline for the treatment of Lyme neuroborreliosis. Scand J Inf Dis 2005; 37: $449-454$

2 Fauser S, Stich O, Rauer S. An unusual case of tick borne encephalitis with isolated myeloradiculitis. J Neurol Neurosurg Psychiatry 2007; 78: 909-910

3 Halperin JJ, Shapiro S, Logigian E, Belman A, Dotevall L, Wormser G, Krupp L, Gronseth G, Bever C. Practice parameter: Treatment of nervous system Lyme disease (an evidence-based review). Neurology 2007; 69: 91 - 102

4 Rupprecht TA, Koedel U, Angele B, Fingerle V, Pfister HW. Cytokine CXCL13 - a possible early CSF marker for neuroborreliosis. Nervenarzt 2006; 77: $470-473$

\section{Korrespondenzadresse}

Prof. Dr. Reinhard Kaiser

Ärztlicher Direktor Neurologische Klinik

Klinikum Pforzheim $\mathrm{GmbH}$

Kanzlerstraße 2-6

75175 Pforzheim

Telefon: 07231/969-2602

Telefax:07231/969-2911

E-mail: RKaiser@klinikum-pforzheim.de 